\title{
Monte Carlo study of secondary electron production from gold nanoparticle in proton beam irradiation
}

\author{
Junfang Gao, Yuanshui Zheng \\ Department of Medical Physics, ProCure Proton Therapy Center, Oklahoma City, Oklahoma, USA.
}

Received January 07, 2014; Revised March 11, 2014; Accepted March 20, 2014; Published Online March 27, 2014

\section{Original Article}

\begin{abstract}
Purpose: In this study, we examined some characteristics of secondary electrons produced by gold nanoparticle (NP) during proton beam irradiation. Method: By using the Geant4 Monte Carlo simulation toolkit, we simulated the NP at the range from radius ( $\mathrm{r}$ ) of $17.5 \mathrm{~nm}, 25 \mathrm{~nm}, 35 \mathrm{~nm}$ to $\mathrm{r}=50 \mathrm{~nm}$. The proton beam energies used were $20 \mathrm{MeV}, 50 \mathrm{MeV}$, and $100 \mathrm{MeV}$. Findings on secondary electron production and their average kinetic energy are presented in this paper. Results: Firstly, for NP with a finite size, the secondary electron production increase with decreasing incident proton beam energy and secondary buildup existed outside NP. Secondly, the average kinetic energy of secondary electrons produced by a gold NP increased with incident proton beam energy. Thirdly, the larger the NP size, the more the secondary electron production. Conclusion: Collectively, our results suggest that apart from biological uptake efficiency, we should take the secondary electron production effect into account when considering the potential use of NPs in proton beam irradiation.
\end{abstract}

Keywords: Gold nanoparticle; Secondary electron production; Proton

\section{Introduction}

Some recent studies tried to explore the potential application of gold nanoparticles (NPs) in various therapeutic and diagnostic physics. ${ }^{1-6}$ Due to their high atomic number $(Z)$, thus, high photoelectric coefficient, the gold NPs have been investigated as dose enhancement agents in radiotherapy. ${ }^{1-3} \mathrm{Be}-$ cause of their high photon stability and high quantum yield, they have been examined for the use as contrast agents in medical imaging. ${ }^{4-6}$ Furthermore, it has been shown that a gold foil surface enhances relative biological effectiveness (RBE) because of gold's high secondary electron production efficiency, ${ }^{7}$ so it can be expected that the surface of a gold NP will cause a similar enhancement.

The essential of all gold NP studies in radiological physics are related to its specific characteristic of secondary electrons produced by irradiation. In photon therapy and diagnostic

Corresponding author: Junfang (Jeff ) Gao, PhD; Department of Medical Physics, ProCure Proton Therapy Center, Oklahoma City, Oklahoma, USA. Email: gao.junfan@yahoo.com

Cite this article as:

Gao J, Zheng Y. Monte Carlo study of secondary electron production from gold nanoparticle in proton beam irradiation. Int $J$

Cancer Ther Oncol 2014; 2(2):02025. DOI: 10.14319/ijcto.0202.5 imaging, photoelectric and Compton scattering effects are dominant in the secondary electron production. In proton therapy, proton induced ionization play dominant role in secondary electron production. The mechanism of proton induced ionization has been depicted in early pioneer literatures. $^{8-9}$ Here we just brief the picture of this interaction. When a proton approaches an atom, due to the opposite charge of proton and orbital electron, orbital electron will be pulled out from atom. After a proton passes through the electron cloud, some orbital electron still keep following proton and leave atom. Only when the proton energy is low enough and its speed is close to the speed of orbital electron, orbital electron can be captured by the passing protons (different from the nuclear electron capture). This electron capture is only dominant at low energy (less than $100 \mathrm{keV}) .{ }^{10}$

As a proton passes through a gold NP, secondary electrons are produced both inside and outside the NP. Because of the high electron volume density (numbers/volume) inside of the gold NP, more secondary electrons are generated inside the gold NP than outside. However, some of the internal electrons will fly outside the NP if their energies are high enough to overcome the surface work function. Detailed information about the secondary electron production distribution is thus crucial in microdosimetry. 
In this paper, we examined the characteristics of secondary electrons produced by a single gold NP during proton beam irradiation using the Geant4 Monte Carlo simulation toolkit. The aim of this paper is to study the effects of proton beam energy and NP size on secondary electron production. Regarding the highest energy in our clinical use is about 250 $\mathrm{MeV}$ and the highest energy contribution to $250 \mathrm{MeV}$ pristine peak is about $100 \mathrm{MeV}$, we investigated proton beams with energy of $20 \mathrm{MeV}, 50 \mathrm{MeV}$, and $100 \mathrm{MeV}$. Upon better biological uptake efficiency from literatures, we chose the NP size from $17.5 \mathrm{~nm}, 25 \mathrm{~nm}, 35 \mathrm{~nm}$ to $50 \mathrm{~nm}$ in radius respectively. We hope this study also provide some fundamental information on our future research on gold fiducial marker which has been broadly used in the proton clinics. To author's knowledge, this is first detail study on the gold nanopaticles interaction with therapeutic proton beam. The information achieved from this study will be a guide for possible future dosimetry calculation on nanoparticle clinical application.

\section{Methods and Materials}

The Geant4. 8.1 simulation package ${ }^{17-18}$, which has been calibrated and used in our previous research ${ }^{11}$, is used in this study. The Monte Carlo simulation geometry used here to examine a proton beam's interaction with a single gold NP is shown in Figure $1 \mathrm{~A}$ spherical gold NP was placed at the center of a cubic water phantom $\left(40 \times 40 \times 40 \mu \mathrm{m}^{3}\right)$; the water phantom itself was positioned at the center of a cubic air world volume $\left(80 \times 80 \times 80 \mu \mathrm{m}^{3}\right)$. All particles were tracked only inside the world volume. Figure 1 shows one dimension of the simulation geometry setup. The uniform proton beam was generated using a circular plate whose diameter was called beam width here. In all our simulations, the total number of protons was $10^{6}$. The circular plate was placed 150 $\mathrm{nm}$ away from the center of the gold NP.

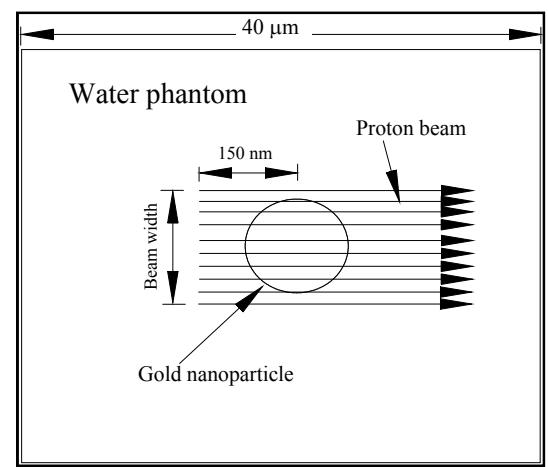

FIG. 1: The sketch of the Monte Carlo simulation setup.

To study the influence on secondary electron from proton energy, we chose the energy of $20 \mathrm{MeV}, 50 \mathrm{MeV}$ and 100 $\mathrm{MeV}$. In the proton therapy, the highest energy of proton beam is $250 \mathrm{MeV}$. The highest proton energy contributed to $250 \mathrm{MeV}$ beam pristine Bragg Peak is about $100 \mathrm{MeV}$ for aperture of $20 \times 20 \mathrm{~cm}^{2}$ as shown in Figure 2. For lower energy beam pristine Bragg peaks, the pristine Bragg peak starting energy will be below $100 \mathrm{MeV}$. In proton therapy only Bragg peak is clinical significant because Spread Out Bragg Peak (SOBP) is comprised of numerous Bragg peaks. SOBP is the supposition of many Bragg peaks which corresponds to different incident energetic proton beam. Figure 2 show only Bragg peak for one incident energy (250 MeV) beam. Any dose enhancement or Relative Biology Effect (RBE) enhancement of NP application should occur only in SOBP region. Proton energies of $20 \mathrm{MeV}, 50 \mathrm{MeV}$, and 100 $\mathrm{MeV}$ cover most of the SOBP region and were used in our investigation.

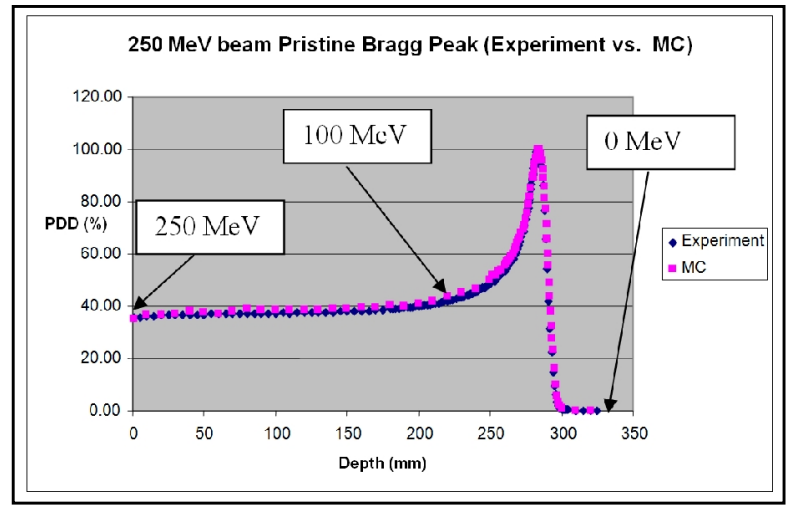

FIG. 2: The calculated and the measured $250-\mathrm{MeV}$ proton pristine Bragg peak.

To quantitatively analyze secondary electron distribution of single NP, we scored the number and kinetic energy of secondary electron by Sensitive Detector (SD) which was associated to a tracking geometry. ${ }^{12}$ The tracking geometry was the whole world volume which contained water phantom, NP and proton beam. Then the SD was associated to a virtual readout geometry which can have different dimensions from real tracking geometry. In this paper, the SD was 40 concentric spherical shells with equal thickness as shown in Figure 3. The smallest SD is a sphere of diameter $5 \mathrm{~nm}$. The thickness of each SD is $5 \mathrm{~nm}$. The number and average kinetic energy of secondary electrons in each shell detector were scored both inside and outside of NP.

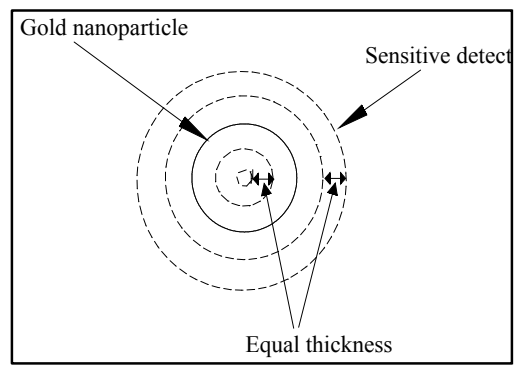

FIG. 3: The sketch of sensitive detector (SD). Sensitive detector shells are in dashed lines. The first sensitive detector is a dashed sphere. Gold NP is in solid line. 
To determine how NP size affects secondary electron production and its average kinetic energy distribution, we chose NP size based on others' studies on NP cellular uptake efficiency. For instance, Chithrani et al. ${ }^{13-14}$ reported that gold NPs with a diameter of $50 \mathrm{~nm}$ had the highest uptake efficiency by mammalian cells. El-Sayed et al. ${ }^{15}$ found that gold NPs with an average diameter of $35 \mathrm{~nm}$ had the highest uptake efficiency by oral epithelial cells. However, in Hiller and Albrecht's ${ }^{16}$ quantitative study of colloid gold distribution in mice, they showed that smaller NPs $(d=4 \mathrm{~nm})$ were more readily taken up by the gastrointestinal tract than larger particles $(\mathrm{d}=10 \mathrm{~nm}, 28 \mathrm{~nm}$, and $58 \mathrm{~nm})$. All these studies indicated that different type of cells had different size preference while they absorb gold NPs. These studies suggested that the NP geometry should be taken into account seriously when we explore the use of gold NPs in radiotherapy. This study will only focus on NP with radius of $17.5 \mathrm{~nm}, 25 \mathrm{~nm}$, $35 \mathrm{~nm}$, and $50 \mathrm{~nm}$.

In the physics interaction, three categories process were executed in the simulation. They are Decay model, Electromagnetic physics model and hadronic physics model. In the electromagnetic physics model, Low-energy physics package were mainly used in delineating photons, electrons and ions related physics process. This is different from Jarlskog and Paganetti's selections ${ }^{22}$ in which the standard electromagnetic physics package is found better to be used to simulate $160 \mathrm{MeV}$ proton's interaction than low energy package. The main reason is that the current study focused on the low proton energy of $20 \mathrm{MeV}, 50 \mathrm{MeV}$ and $100 \mathrm{MeV}$ and the average secondary electron kinetic energy is about 2 to 4 $\mathrm{keV}$. They were listed as following:

\section{1) Decay model.}

\section{2) Electromagnetic physics model:}

\section{Photons:}

photon-epdl___ Livermore-based models (Low-energy package)

\section{Electrons:}

electron-eedl___ Livermore-based models (Low-energy package)

Positrons:

positron-standard____ Standard models (Standard package)

Charged hadrons, ions:

ion-LowE___ ICRU49-based models (Low-energy package)

ion-LowE-ziegler1977___ Ziegler77-based models (Low-energy package)

ion-LowE-ziegler1985__ Ziegler85-based models (Low-energy package)

ion-LowE-ziegler2000___ Ziegler2000-based models (Low-energy package)

\section{Muons:}

muon-standard___ Standard models (Standard package)

\section{3) Hadronic physics model:}

\section{Proton:}

proton-hadronic____Standard model

proton-precompound___ Precompound model (default evaporation)

proton-precompoundFermi___ Precompound model (default evaporation, Fermi break-up)

proton-precompoundGEM___ Precompound model (GEM evaporation)

In above total cross section eedl database, the energy of electron is ranged from $250 \mathrm{eV}$ to $100 \mathrm{GeV}$. In ICRU49-based models ion-LowE, the knock-on electron cross section was well described from $10 \mathrm{eV}$ to $0.1 \mathrm{TeV}$ in 360 bins. 


\section{Results and Discussion}

Our first finding is that, for NPs with a finite size (Here $r=$ $35 \mathrm{~nm}$ ), the secondary electron production was proportional to the incident proton beam energy and outside the NP an obvious minima indicated electron secondary buildup existed. Figure 4 shows the secondary electron distribution corresponding to different incident proton energies. In this study, the beam width of $120 \mathrm{~nm}$ and NP radius of $35 \mathrm{~nm}$ (diameter is $70 \mathrm{~nm}$ ) were considered. The thin vertical line in all following figures indicated the surface of gold NP. From Figure 4 the proton beam with energy of $20 \mathrm{MeV}$ has highest secondary electron production both inside and outside NP. The $100 \mathrm{MeV}$ proton beam has the lowest electron production. The total number of secondary electrons produced by proton beam increased with decreasing proton beam energy from $100 \mathrm{MeV}$ to $20 \mathrm{MeV}$. The shape of each distribution is very similar. Inside the NP, the secondary electron number increased in quadratic order with the increase of distance from the center of NP. Because the beam width is much wider than the size of single NP, secondary electrons are generated from the ionization of proton impact interactions with gold atom uniformly inside the NP. The secondary electron density is approximately a constant. This quadratic increase was caused by the volume increase following $\left\{\frac{4 \pi}{3}\left[d^{3}-(d-\text { thickness })^{3}\right]\right\}$ of each sensitive detector shell, where $d$ is the distance of SD shell from center of NP.

The center SD has least volume and collects least electrons. The SD closer to surface has larger volume and it can collect more electrons. The peak which was right inside the NP surface line indicated that most secondary electrons were restricted by surface work function. Outside the NP, secondary electrons are from both electrons scattered from inside NP and electrons produced in the water. Scattered electrons with average kinetic energy about $2 \mathrm{keV}$ to $3 \mathrm{keV}$ (from Figure 6) won't travel too far and they only travel in the NP peripheral region. These electrons are expected to be clinically significant and cause some dose enhancement. A minima can be seen clearly outside NP surface for all energies. Our interpretation about this minima lies in that the scattered electrons from inside NP continuously slow down and stop at some point where the secondary electrons produced from water still haven't achieve electron equilibrium. The electron scattered from inside to outside is more than those scattered from outside to inside. Borrowing a "word" from photon world, we call this is secondary electron buildup region. In the distal region from the NP surface, a plateau area was caused mostly by the secondary electron produced from water and indicated an electron equilibrium existed. In water the secondary electrons production are much less than those inside NP. The uncertainty of the number secondary electron of $20 \mathrm{MeV}, 50 \mathrm{MeV}$, and 100 $\mathrm{MeV}$ are $0.5 \%, 0.7 \%$, and $1.09 \%$ respectively.

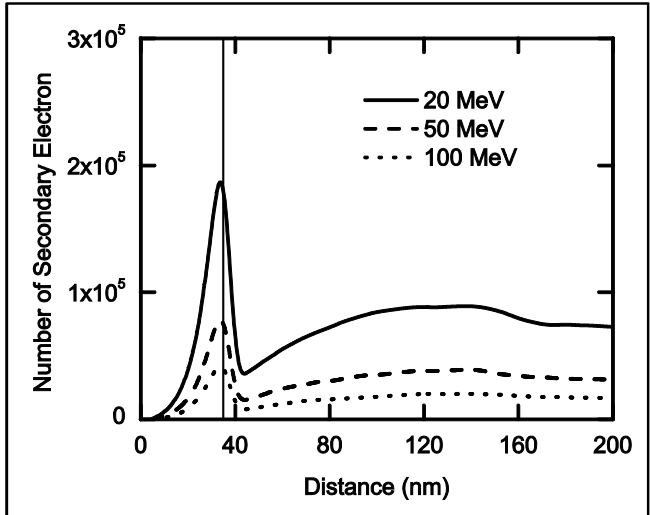

FIG. 4: Number of secondary electrons produced by the gold NP with a radius of $35 \mathrm{~nm}$ undergoing irradiation with $20-\mathrm{MeV}$, $50-\mathrm{MeV}$, and $100-\mathrm{MeV}$ proton beams. The vertical thin line represents the surface of the NP.

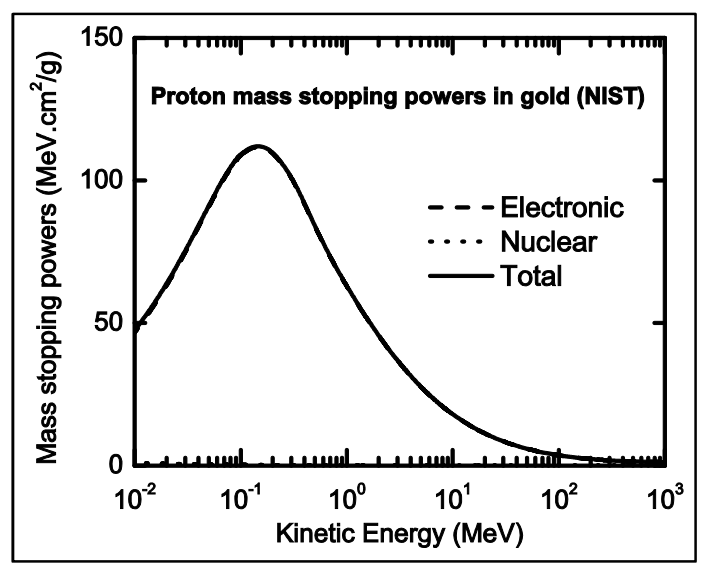

FIG. 5: Proton mass stopping power in gold. The mass stopping power of electronic ionization (dashed line) and that of nuclear interaction (dotted line) are shown along with the total mass stopping power. Because the contribution of the nuclear interaction to the total mass stopping power is very small (dotted line coincides with the horizontal axis), the electronic stopping power is almost the same as total stopping power.

To understand phenomena inside NP above, we can also reference the proton mass stopping power in gold. Figure $\mathbf{5}$ is the diagram of proton mass stopping power calculated from NIST website. ${ }^{19}$ The dominant part of proton mass stopping power is contributed from electronic ionization as the proton energy is less than $100 \mathrm{MeV}$. The nuclear interaction contribution is pretty low (dotted line coincides with horizontal axis). So the electronic contribution is almost the same as the total mass stopping power (dashed line coincides with solid line). From Figure $\mathbf{5}$ lower energy proton has a higher mass stopping power in gold material, and so higher electronic ionization probability. Thereafter, the low energy proton beam can produce more secondary electrons than high en- 
ergy proton beam inside gold NP. This is pretty much what we have seen in Figure 4. As to the outside of gold NP, similar physics as inside god NP except in water materials. Therefore, we saw highest secondary electron production in $20 \mathrm{MeV}$ proton beam and lowest secondary electron production in $100 \mathrm{MeV}$ beam while the $50 \mathrm{MeV}$ is in the between in Figure 4.

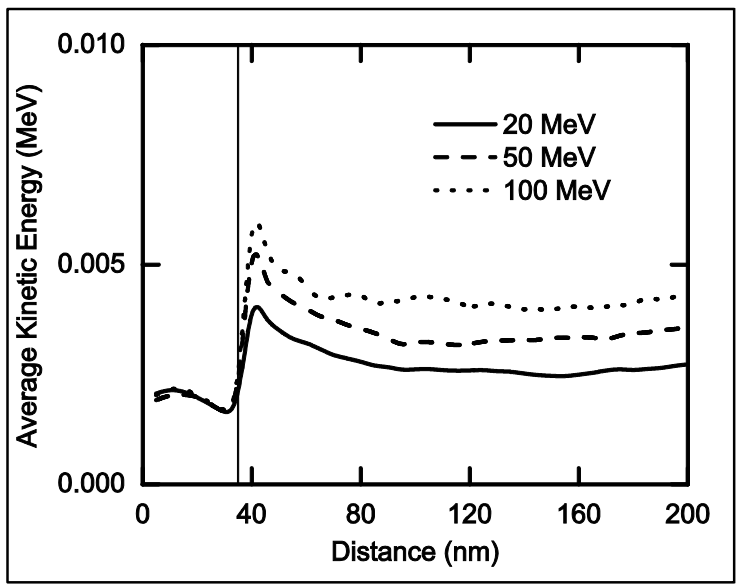

FIG. 6: Average kinetic energy distribution of secondary electrons around the gold NP with a radius of $35 \mathrm{~nm}$ during irradiation with $20-\mathrm{MeV}, 50-\mathrm{MeV}$, and $100-\mathrm{MeV}$ proton beams. The thin vertical line represents the surface of the NP. As shown, inside the NP, the average kinetic energy for each proton beam energy is roughly the same (i.e., the three lines appear to overlap).

Figure 6 showed our second finding about the average kinetic energy of secondary electrons. In this study, we calculated the average kinetic energy as the total energy of electrons in each SD shell divided by the total number of electrons in that shell. We found that outside of the gold NP, the average kinetic energy of secondary electrons produced by the $100-\mathrm{MeV}$ proton beam was higher than that of those produced by the $50-\mathrm{MeV}$ proton beam; and the average kinetic energy of secondary electrons generated by the $50-\mathrm{MeV}$ proton beam was higher than those generated by the $20-\mathrm{MeV}$ proton beam. The equal space between the distributions indicates that the average kinetic energy of secondary electrons was roughly linearly proportional to incident proton beam energy. Inside the NP, average kinetic energy of secondary electrons was almost the same for the three proton beams. An obvious peak existed outside the NP and initiated the discrepancy in the rest distal plateau region for all three energies.

Our interpretation about the second finding is based on a semi-empirical formula ${ }^{15}$ of secondary electron maximum energy $\left(E_{M}\right)$,

$$
E_{M} \propto \frac{4 T}{\lambda},
$$

Where $T$ is the kinetic energy of the incident protons and $\lambda$ $=1836$. From the formula above, the maximum energy of secondary electrons is linearly proportional to the incoming proton kinetic energy. We can approximately hold this as true for average energy of secondary electrons. This is depicted from the track structure calculation performed by Uehara et al. ${ }^{10}$ Outside NP in Figure 6, three lines are about equal spaced and corresponding to the $30 \mathrm{MeV}$ and $50 \mathrm{MeV}$ energy gap between $20 \mathrm{MeV}, 50 \mathrm{MeV}$ and $100 \mathrm{MeV}$. We think this is caused by the linearly proportional relation above. Inside the gold NP, the average kinetic energy is lower than those of outside in water and they are about same for different energy proton beams. The physics inside the NP is still not very clear to us. The following is just our speculation. The first ionization energy of gold atoms is about $9 \mathrm{eV}$, and the first ionization energy of water molecules is $12.6 \mathrm{eV}$. The gold atom is easier than water molecular to be ionized by proton beam induction. Thus more secondary electrons are produced inside than outside NP as shown in Figure 4. But the mass stopping power for proton is higher in gold than in water and the electrons lose energy faster in gold than in water. The secondary electrons travel shorter in gold than in water. On average, even the total number of secondary electrons is higher, but the kinetic energy inside gold is lower than that outside NP in water. This is what we saw in Figure 6. We are puzzled why the kinetic energy is about same for those secondary electrons corresponding to different proton energy beams. We wonder if this is related to the unique energy structure of gold NP. From Figure 6, some high energy peaks occurred at position corresponding to the minima of Figure 4. At these minima point, the number of scattered electrons with lower energy from inside NP decreased dramatically and the high energy electrons produced in the water started to increase but didn't have chance to lose a lot energy. Then on average, the kinetic energy for secondary electron at this point is the highest. So we have a peak at this point.

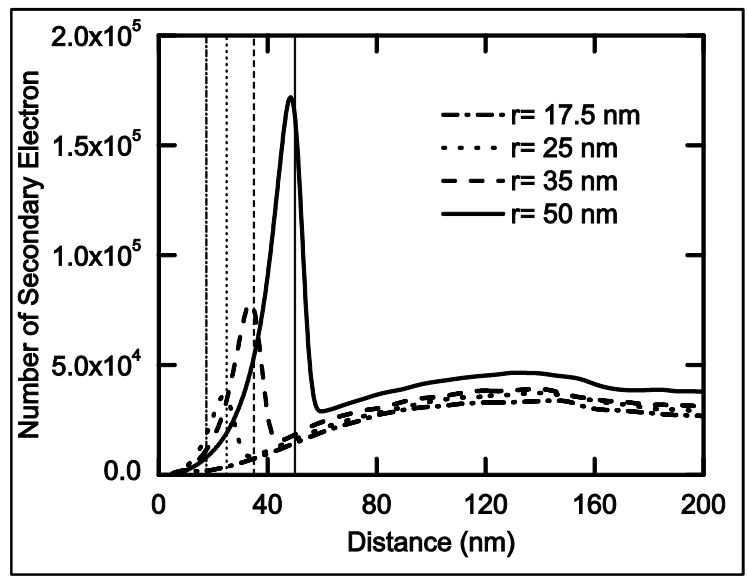

FIG. 7: Number of secondary electrons produced by NPs of different sizes during irradiation with a $50 \mathrm{MeV}$ proton beam. The thin vertical lines represent the surface of each NP.

Figure 7 presents the third finding from our simulation. It shows that the number of secondary electrons produced inside NP increased with NP size. As to the outside, regard- 
ing the statistic uncertainty of secondary electron corresponding to $50 \mathrm{MeV}$ proton beam, there is no significant discrepancy in the plateau region among different size NPs. We selected NP size based on the biological uptake efficiency reported in the literatures as we mentioned before. Here, we chose the radius with $\mathrm{r}=17.5 \mathrm{~nm}, 25 \mathrm{~nm}, 35 \mathrm{~nm}$, and 50 $\mathrm{nm}$. The incident proton beam energy was $50 \mathrm{MeV}$, with beam width of $120 \mathrm{~nm}$ and fluence of $354 \mathrm{~nm}^{2}$. In general, the secondary electron distribution shapes are similar for different size NPs. The peak height of NP with $r=50 \mathrm{~nm}$ is about 2.2 times that of NP with $\mathrm{r}=35 \mathrm{~nm}$. The peak height of NP with $r=35 \mathrm{~nm}$ is about 2.0 times that of NP with $r=$ $25 \mathrm{~nm}$. For NP with $\mathrm{r}=17.5 \mathrm{~nm}$, the peak close to the NP's surface was very small because a small number of protons can traverse the surface region of the NP. When the size increased, the peak position shifted to right and but always on the NP surface.

From physics aspect, the larger gold NP has more secondary electron production inside the NP. Outside the NP, water phantom has pretty much same production for the $50 \mathrm{MeV}$ protons regardless of the NP size. Inside the NP, the four distributions are following the quadratic increase trend with similar shape. We think this might be caused by some effect related to surface property. According to ICRU Report 5520, the largest NP had the highest peak because of the charged-state effects in the gold. When charged particles penetrate a solid, the charge equilibrium is broken by secondary electrons generated by the charged particles. This charge un-equilibrium region may extend several nanometers. Because of the surface barrier, this type of region commonly occurs close to the NP's surface; thus, larger NPs with larger surface area will have larger un-equilibrium regions inside the NP than smaller NPs. Electrons in the un-equilibrium region will migrate to the surface and eventually escape the gold NP. Higher energy electrons easily traverse the surface barrier and fly out of the NP. These escaping electrons, in turn, contribute to the average kinetic energy of secondary electrons produced outside the NP, which is higher in larger NPs than smaller one. We conclude that more secondary electrons are produced by the larger NPs than the smaller NPs during proton irradiation. This non-equilibrium region helps to produce more secondary electrons than the same region if it is not in the surface of other size.

\section{Conclusions}

In this study, we examined, for the first time, the characteristics of secondary electrons produced by gold NPs during proton beam irradiation using the Geant4 Monte Carlo simulation toolkit. We found that for NPs with a finite size, the secondary electron production was linearly proportional to the incident proton beam energy. We also found that the average kinetic energy of secondary electrons produced by a gold NP increased with incident proton beam energy. In size effect, we concluded that the larger the NP, the higher the secondary electron production. Collectively, our results suggest that apart from biological uptake efficiency, we should take the surface secondary electron production into account when considering the use of NPs in radiotherapy.

\section{Conflict of interest}

The authors declare that they have no conflicts of interest. The authors alone are responsible for the content and writing of the paper.

\section{References}

1. Herold DM, Das IJ, Stobbe CC, et al. Gold microspheres: a selective technique for producing biologically effective dose enhancement. Int J Radiat Biol 2000; 76: 1357-64.

2. Hainfeld JF, Slatkin DN, Smilowitz HM. The use of gold nanoparticles to enhance radiotherapy in mice. Phys MedBíD2004; 49: N309-15.

3. Cho SH. Estimation of tumour dose enhancement due to gold nanoparticles during typical radiation treatments: a preliminary Monte Carlo study. Phys Med Biol2005; 50: N163-73.

4. Sharma P, Brown S, Walter G, et al. Nanoparticles for bioimaging. Adv Colloid Interface Sci 2006; 123-126: 471-85.

5. Shim SY, Lim DK, Nam JM. Ultrasensitive optical biodiagnostic methods using metallic nanoparticles. Nanomedicine (Lond) 2008; 3: 215-32.

6. Skrabalak SE, Au L, Lu X, et al. Gold nanocages for cancer detection and treatment. Nanomedicine (Lond) 2007; 2: 657-68.

7. Regulla D, Schmid E, Friedland W, et al. Enhanced values of the $\mathrm{RBE}$ and $\mathrm{H}$ ratio for cytogenetic effects induced by secondary electrons from an X-irradiated gold gurface. Radiat Res 2002; 158: 505-515.

8. Rudd ME, Kim YK, Madison DH, et al. Electron Production in Proton Collisions: Total Cross Sections. Rev Mod Phys 1985; 57:965-94.

9. Rudd ME, Kim YK, Madison DH, et al. Electron production in proton collisions with atoms and molecules: energy distributions. Rev Mod Phys 1992; 64: 441-90.

10. Uehara S, Toburen LH, Nikjoo H. Development of a Monte Carlo track structure code for low-energy protons in water. Int J Radiat Biol 2001; 77:139-54.

11. Zhang SX, Gao J, Buchholz TA, et al. Quantifying tumor-selective radiation dose enhancements using gold nanoparticles: a monte carlo simulation study. Biomed Microdevices 2009; 11: 925-33. 
12. http://geant4.web.cern.ch/geant4/UserDocumentati on/UsersGuides/ [Accessed date: March 1, 2008 ]

13. Chithrani BD, Ghazani AA, Chan WC. Determining the size and shape dependence of gold nanoparticle uptake into mammalian cells. Nano Lett 2006; 6: 662-8.

14. Chithrani BD, Chan WC. Elucidating the mechanism of cellular uptake and removal of protein-coated gold nanoparticles of different sizes and shapes. Nano Lett 2007; 7:1542-50.

15. El-Sayed IH, Huang X, El-Sayed MA. Surface plasmon resonance scattering and absorption of anti-EGFR antibody conjugated gold NPs in cancer diagnostics: application in oral cancer. Nano Lett 2005; 5: 829-34.

16. Hillyer JF, Albrecht RM. Gastrointestinal persorption and tissue distribution of differently sized colloidal gold nanoparticles. I Pharm Sci 2001; 90:1927-36.
17. Agostinelli S, Allison J, Amako K, et al. Geant4-a simulation toolkit. Nuclear Instruments and Methods in Physics Research Section A: Accelerators, Spectrometers, Detectors and Associated Equipment. 2003; 506: 250-303.

18. Allison J, Amako K, Apostolakis J, et al. Geant4 developments and applications. Nuclear Science, IEEE Transactions on 2006; 53: 270-8.

19. http://physics.nist.gov/PhysRefData/Star/Text/PST AR.html [Accessed date: February 1, 2014 ]

20. ICRU report 55. Secondary electron spectra from charged particle interactions. International commission on radiation units and measurements, Bethesda Maryland, 1996.

21. Polf JC, Bronk LF, Driessen WH, et al. Enhanced relative biological effectiveness of proton radiotherapy in tumor cells with internalized gold nanoparticles. Appl Phys Lett 2011; 98:193702.

22. Jarklskog CZ, Paganetti H. Physics settings for Using the Geant4 Toolkit in Proton Therapy. Nuclear Science, IEEE Transactions on 2008; 55: 1018-25. 